\title{
IL DIAVOLO IN CORPO: UNA LETTURA DEL Decameron di Giovanni Boccaccio
}

\author{
THE DEVIL IN THE FLESH: \\ A READING OF BOCCACCIO'S DECAMERON
}

Andrea Lombardi

Universidade Federal do Rio de Janeiro

Rio de Janeiro, RJ, Brasil

Palavras-chaves: ironia, erotismo, desconstrução, clássicos (releitura dos), Decameron, Giovanni Boccaccio, arquitetura,
Parole-chiave: ironia, erotismo, decostruzione, classici (rilettura dei), Decameron, Giovanni Boccaccio, architettura.

Keywords: irony, eroticism, deconstruction, classics (rereading of), Decameron, Giovanni Boccaccio, architecture

\section{Resumo}

Considerar o Decamerão apenas como um "clássico" não faz justiça à sua estrutura narrativa geométrica e complexa. Pois cada um de seus aspectos revela o potencial subversivo de sua narrativa. As cem novelas junto com sua moldura, que descreve a epidemia da Peste Negra em 1348, fazem dele o primeiro livro orgânico da narrativa ocidental, um texto com sua arquitetura peculiar. Porém, uma leitura cuidadosa pode revelar a existência de uma nova novela, a de número 101 (na Introdução à Quarta Jornada): o que representa a crise final da estrutura fechada, de uma arquitetura planejada. Ciappelletto, protagonista da primeira novela, torna-se Santo, de "pior homem do mundo" que era; enquanto Griselda, heroína da última das cem novelas mostra como suas hiperbólicas virtudes se transformam em cinismo cruel. Assim, o Decamerão cria seu próprio futuro, por representar uma mímese abrangente de sua época e, ao mesmo tempo, realizar uma ruptura irônica radical, ou elusiva: modelo da comédia renascentista e também um paradigma hermenêutico do uso da ironia. Talvez possamos considerar o Decamerão uma intrigante resposta à pergunta atual sobre a natureza do contemporâneo.

\section{Riassunto}

Considerare il Decameron esclusivamente un "classico" non rende giustiza alla sua struttura narrativa geometrica e complessa. Poiché ognuno dei suoi aspetti rivela il potenziale sovversivo della sua macchina narrativa. Le cento novelle con la loro cornice, che descrive l'epidemia della peste nera del 1348, ne fanno il primo libro organico della narrativa occidentale: un testo con

\begin{abstract}
Considering Boccaccio's Decameron only as a "classic" does not render justice to its narrative structure, geometric and complex. For each of its aspects reveals the subversive potential of its narrative. Both the hundred novels and the frame, evoking the Black Death epidemic in 1348, make it into the first organic book in the Literature of the West, a text with a planned architecture. A
\end{abstract}


una architettura peculiare. Una lettura attenta, però, può individuare una nuova novella, quella di numero 101 (nell'Introduzione alla quarta giornata): ciò che rivela la crisi finale della struttura chiusa, dell'architettura pianificata. Ciappelletto, protagonista della prima novella, diviene Santo da "peggiore uomo del mondo" che era; mentre Griselda, eroina dell'ultima delle cento novelle, mostra che la sua iperbolica virtù si trasforma in cinismo crudele. Così il Decameron crea il proprio futuro, rappresentando una mimesi ampia della sua epoca e, allo stesso tempo, avviando una rottura radicale ironica, o meglio elusiva: modello della commedia rinascimentale e dell'ermeneutica, nell'uso radicale dell'ironia. Possiamo forse considerare il Decameron una risposta istigante alla domanda attuale sulla natura di ciò che è contemporaneo. careful reading, however, can identify a new novella, this one whose number is 101 (in the Introduction of the forth Day): a striking lack of narrative closure and of its architecture. Ciappelletto, the protagonist of the first novel, is transformed from the "worst man in the world" into a hypothetical Saint and Griselda, the heroine of last novel, shows her hyperbolic virtues turning in cruel cynicism. The Decameron thus creates its own future, because it represent an exaustive Mimesis of his age, and at the same time, creates a radical ironic break, or rather an elusive one; becoming a model for the Renaissance comedies and a hermeneutic paradigm for the use of irony. From that point of view, the Decameron could be considered an intriguing response to the current debate on what is contemporary.

Capita sempre più di rado d'incontrare persone che sappiano raccontare qualcosa come si deve...

Walter Benjamin, Il Narratore

La cópula, l'unione di due organismi unicellulari, ha l'effetto di tenere in vita e ringiovanire entrambi Sigmund Freud, Al di là del principio del piacere

Il diavolo in corpo, titolo di un racconto dell'inizio del sec. XX di R. Radiguet e di un film di Marco Bellocchio, originalmente deriva da una frase contenuta nella novella di Masetto del Decameron (III giornata, $1^{\text {a }}$ novella), scritto presumibilmente fra il $1349 \mathrm{e}$ il 1351, immediatamente dopo gli effetti della grande epidemia di peste nera che ha decimato l'Europa. Il testo originale del Decameron è diviso in dieci capitoli o giornate, in cui una brigata di dieci giovani (sette donne e tre uomini) decide di sfuggire agli orrori del flagello e, in un luogo appartato, a poca distanza dal centro della città, 
* (BOCCACCIO, Giovanni. Decameron. V. Branca (org.). Torino: Einaudi, 1992: 9.) durante due settimane (per osservare i giorni di riposo tradizionali e religiosi) si racconta vicendevolmente un totale di cento novelle. Ogni giornata ha un tema determinato (con alcune eccezioni), che ruota attorno a una variazione dei concetti di amore, fortuna, beffa e motto di spirito, che permea tutto il testo. I dieci narratori, ciascuno con una precisa personalità, commentano vivacemente le novelle appena raccontate, nel corso delle giornate, ognuna retta da un "re" o una "regina", scelti fra i dieci narratori. Il libro è dedicato alle donne al posto della tradizionale dedica alle Muse; il suo fine dichiarato è quello di dare voce alla loro voce, un manuale dei casi dell'amore e della vita. Per ottenere un utile consiglio:

le già dette donne, che quelle leggeranno, parimenti diletto delle sollazzevoli cose in quelle mostrate e utile consiglio potranno pigliare, in quanto potranno cognoscere quello che sia da fuggire e che sia similmente da seguitare. ${ }^{1 *}$

Il Decameron può essere considerato un libro cinematografico, basato com'è tutto sul movimento. La sua struttura interna si fonda su una sequenza di verbi e di azioni, la cui base grammaticale è la còpula, nel doppio senso di legamento verbale e, anticipando la tematica erotica, l'amplesso. La scelta della lingua volgare non dovrà essere giustificata, così come era stato necesario al tempo di Dante (con il suo testo teorico, scritto in latino: De vulgari eloquentia). Sarà naturale, come naturale è la legge della cópula. Boccaccio non avrà più l'esigenza di mostrare una via maestra, un inquadramento definitivo, un mondo alla luce della provvidenza divina, ragione per cui è stato accusato, nel sec XIX dal critico romantico Francesco De Sanctis (Storia della letteratura italiana), e nel sec. XX da Erich Auerbach (Mimesis) di scarsa dirittura etica o di fiacchezza stilistica e dietro al suo "scarso sentimento preumanistico" si sente un dubbio sull'autorità morale di Boccaccio, evidentemente in conronto a Dante. ${ }^{2}$ Si può obiettare qui che la pro-

\footnotetext{
1 "As damas que essas páginas lerem poderão encontrar ensinamentos úteis nos casos agradáveis que aparecem em tais histórias e receber conselhos úteis quanto à atitude que convém ou não adotar na vida." (BOCCACCIO, Giovanni. Decameron. U. Tavares Rodrigues (trad.). Belo Horizonte: Crisalida, 2005: 14.)

2 "Exatamente onde o Boccaccio tenta penetrar na problemática do trágico, revela-se a obscuridão e a incertezade seu sentimento pré-humanístico. O seu realismo libre, rico, magistral no domínio dos fenómenos, plenamente natural dentre dos limites do estilo médio, torna-se fraco e superficial não apenas toca o problemático e o trágico [...] O sentimento mundano dos homens como Boccaccio era ainda incerto e instável para oferecer uma base que, como quela interpretação, permi-
} 
fonda morale del testo, in contrasto con una morale tradizionale, si esprime nell'impegno a riprodurre la totalità del mondo, con la sua diversificazione libera e cinematografica, compreso il suo lato prosaico, scurrile; senza maschere né censure e, sopratutto (cambiando la prospettiva della lettura), denudare la stessa macchina narrativa e la sua struttura, appena accennate. È possibile pensare che il denudamento del corpo corrisponda a una operazione di smontaggio delle vecchie convenzioni, ampiamente superate dal movimento di un mondo protocapitalista in espansione frenetica. Le novelle stesse, per lo più molto corte, presentano una varietà estrema di motivi e sono ambientate nelle città italiane e europee dell'epoca, nel favoloso Medio Oriente o nell'avventuroso mondo dei mercanti, includendo sempre un numeroso gruppo di partecipanti, con un pluralismo di voci, di commenti, di prospettive che mima un mercato o una fiera del reale. In definitiva, questo diavolo in corpo esprime l'ironia di Boccaccio verso un mondo di credenze allora (come oggi) ancora salde: miti e ipocrisie che il testo contribuisce a smontare.

La prima novella della terza giornata (III, 1), a un terzo del totale, può essere considerata paradigmatica. Si trata della novella di Masetto, in cui il protagonista si presenta come finto sordomuto e diviene l'amante delle otto monache e della badessa di un convento. É nel suo incontro con il vecchio giardiniere, $N u t o$, che la frase sul diavolo in corpo affiora, allusiva, in rapporto allo sviluppo della trama. Nuto si lamenta del carattere delle giovani suore, accenna al suo lavoro faticoso, ma non accenna a commistioni sessuali:

Io lavorava un lor giardino bello e grande e oltre a questo andava alcuna volta al bosco per le legne, attigneva acqua e faceva cotali altri servigetti; ma le donne mi davano sì poco salario, che io non ne poteva appena pur pagare i calzari. E oltre a questo, elle son tutte giovani e parmi ch'ell'abbiano il diavolo in corpo, ché non si può far cosa niuna a lor modo.*3

tisse de ordenar o mundo em seu complexo como realidade, explicá-lo e representá-lo." (AUERBACH, Erich. Mimesis. O realismo na literatura ocidental. Torino: Einaudi, 2000: 247. Grifo meu.)

3 "Eu trabalhava na grande e bela horta das religiosas e, às vezes, ia buscar lenha no bosque, ou ia buscar água, ou fazia outros serviços do mesmo gênero. Mas as freiras pagavam-se tão mal que o dinheiro nem me chegava para os sapatos. Além disso, são todas muito novas e parece que tem o diabo no corpo. Nunca se faz nada ao jeito delas." (BOCCACCIO, Giovanni. Decameron. U. Tavares Rodrigues (trad.). Belo Horizonte: Crisalida, 2005: 159.) 
Come giardiniere, già anziano e padre di numerosi figli venuti alla luce nelle sua carriera di amatore delle monachelle, Masetto ritornerà ricco e felice al suo paese natale. Una novella erotica di un sordomuto, anche se finto, è solo in apparenza un paradosso che mette in luce l'arte del novellare. L'accento iniziale ricade sulla visione (contro l'audizione): totale, impudica, libera, come quando il protagonista Masetto verrà trovato dalla madre badessa tutto scoperto. Uno smascheramento effettivo, un denudamento totale, che mette in luce l'organo sessuale di Masetto:

Masetto, il quale di poca fatica il dì per lo troppo cavalcar della notte... tutto disteso all'ombra di un mandorlo... e avendogli il vento

La scena ribalta una logica confessionale con una forza maggiore di molti discorsi e permette alla letteratura di rovesciare il rapporto di sudditanza rispetto alla pittura (ut pictura poesis), poiché come si lamenterà l'autore del Decameron nelle sue Conclusioni, il grande pittore Giotto fa Cristo maschio e Eva femina. Perché pertanto la letteratura non dovrebbe fare altrettanto, presentare e dipingere il mondo nel suo erotismo? Lo stesso Boccaccio affermerà, dunque, il suo diritto a mettere a nudo la realtà che descrive e stabilisce un'ideale giustapposizione fra letteratura e pittura a lui contemporanea, anticipando le accuse mossegli puntualmente di oscenità:

Saranno per avventura alcune di voi che diranno che io abbia nello scriver queste novelle troppa licenzia usata, sì come in fare alcuna volta dire alle donne e molto spesso ascoltare cose non assai convenienti né a dire né ad ascoltare ad oneste donne...Senza che alla mia penna non dee essere meno d'autorità conceduta che sia al pennello del dipintore, il quale senza alcuna riprensione... fa Cristo maschio e Eva femina...*5

\footnotetext{
4 "Masetto (a quem depois dos excessos da noite, qualquer coisa cansava durante o dia) deitado a dormir à sombra de uma amendoeira... O vento levantara-lhe a frente da camisa, de modo a descobri-lo tudo" (BOCCACCIO, Giovanni. Decameron. U. Tavares Rodrigues (trad.). Belo Horizonte: Crisalida, 2005: 162.)

5 "Algumas de vós dirão que ao escrever as minhas histórias dei rédea solta à licença; que, por exemplo, fiz escutar e dizer às damas o que não é próprio para os ouvidos ou para as bocas de uma mulher honesta...A minha pena pode exigir toda a liberdade que é dada ao pincel do pintor. ... que dá a Adão e a Eva os atributos do seu sexo..." (BOCCACCIO, Giovanni. Decameron. U. Tavares Rodrigues (trad.). Belo Horizonte: Crisalida, 2005: 625.)
} 
L'autore mette l'accento sulla questione dell'autorità, che in rapporto al conflitto fara pittura e poesía è fondamentale. ${ }^{6} \mathrm{Quando}$ Masetto avrà sfruttato tutti i vantaggi del suo essere muto, sollazzandosi con le monachelle e con la badessa, il narratore si mostrerà guarito e apparirà miracolosamente pronto a parlare (cominciò $a$ dire):

s'avvisò che il suo essere mutolo gli potrebbe... in troppo gran danno resultare; e perciò una notte, colla badessa essendo, rotto lo scilinguagnolo, cominciò a dire...* ${ }^{* 7}$

* (Ibidem: 335.)

Nella novella della curiosa adolescente Alibech, il diavolo è il vero protagonista, anche se non si tratta di un essere ultraterreno, ma qualcosa di molto più pragmatico e materiale: "andiamo a rimettere il diavolo in inferno" diviene una curiosa e sconcia forma di alludere al commercio sessuale, fra la curiosa e appetibile Alibech e il monacho Rustico, eremita nel deserto. Il gioco di parole unisce inferno e ninfa nel ninferno. L'atto verrà ripetuto a piacere fino a raggiungere, anche se provvisoriamente, una sensazione di pace. In questo movimento sta un aspetto della sorprendente libertà di un libro che si può considerare una vera utile memoria per il millennio che è appena iniziato. Il testo è profondamente ottimista, allegro, vivace, scostumato, iconoclasta, leggero. Il suo obiettivo dichiarato è servire da consolazione alle pene d'amore, vincere la malinconia, ma anche superare l'orrore della tremenda epidemia di peste. Ma, in questo, non si presenta come un testo didattico, pedante, serio. Sul finire delle novelle il narratore ci rivela che "l'arte può essere dall'arte schernita"* e si accenna a una proliferazione del testo, una sua apertura, che prefigura l'imitazione a cui il modello sarà sottoposto, Dall'imitazione alla moltiplicazione, alla parodia:

se voi ben riguardate, la nostra brigata, già da più altre saputa dattorno, per maniera potrebbe multiplicare. ${ }^{* 8}$

* (BOCCACCIO, Giovanni. Decameron. V. Branca (org.). Torino: Einaudi, 1992 1250.)

\footnotetext{
${ }^{6}$ In particolare, il testo di Lucia Battaglia Ricci, Ragionare nel giardino (Roma: Salerono, 1987), esamina con cura questa ipotesi.

7 "pôs se a pensar que o seu papel de mudo podia, se se prolongasse, causar-lhes grandes e irremediáveis danos. Por isso, uma noite que estava junto da abadessa rompeu o silêncio" (BOCCACCIO, Giovanni. Decameron. U. Tavares Rodrigues (trad.). Belo Horizonte: Crisalida, 2005: 162.)

8 "De resto, olhando as coisas friamente, a nossa brigada é como outras das proximidades." (BOCCACCIO, Giovanni. Decameron. U. Tavares Rodrigues (trad.). Belo Horizonte: Crisalida, 2005: 623.)
} 
* (BOCCACCIO, Giovanni, op. cit., Proemio, Vol I: 8-9.)
L'intreccio profondo fra Amore e Morte (Eros e Tanatos) si riflette nella sua cornice: la descrizione tremendamente realistica della peste e anche in alcune novelle, anticipando sensibilmente l'esempio, anch'esso basato su questa tensione delle Mille e una notte, un testo contemporaneo nella concezione, anche se anonimo. Le novelle, in fondo, potranno essere raggruppate su cinque temi: l'erotismo, la beffa, la morte, la letteratura come cura e, finalmente, un elemento molto importante per la storia della letteratura: la nascita del narratore e, di riflesso, del suo pubblico, già prefigurati nel testo.

Considerato una delle fonti della tradizione narrativa occidentale, a un mitico punto di incontro fra oralità e scrittura, il Decameron mostra, contrariamente a quanto sarebbe logico aspettarsi, una oralità solo simulata e al contrario, enfatizza il suo carattere testuale, il suo essere destinato alla lettura:

intendo di raccontare cento novelle, o favole, o parabole o istorie che dire le vogliamo...le già dette donne, che quelle leggeranno.*

Il testo può variare nel suo genere e, come dirà più tardi (nelle Conclusioni), Boccaccio si presenta solo come un narratore, ma si trata di un iper narratore (oltre ai dieci presentati). È un'epoca in cui si comincia appena a leggere i testi in silenzio (in precedenza le letture, anche individuali, venivano effettuate ad alta voce) e si sta formando un pubblico che, più avanti, sarà il pubblico dei lettori e acquirenti dei libri. Le novelle sono destinate ai lettori intenzionali e non più a ascoltatori casuali. Anche il riso, come la lettura, tenderà a essere silenzioso, punterà all'ironia, nonostante gli elementi comici, trasgressivi, grotteschi siano fondamentalmente teatrali.

\section{La religione dell'erotismo}

Con il Decameron Boccaccio ha scritto una vera e propria grammatica dell'erotismo, una molteplicità di racconti o novelle (un genere nuovo da lui affermato) intrecciati l'uno nell'altro come un rosario, il cui tema centrale e motore è l'eros. Il concetto di novella viene usata nel testo tra l'altro come sinonimo di novità, distrazione (VII, 2), storia non vera, menzogna (VII, 8), chiacchera (VIII, 1). Le ricche e corpose pennellate del testo presentano il nuovo mondo dei mercanti, i nuovi borghesi, abitanti della città, nella loro routine fatta di sessualità, lotta per la sopravvivenza, alimentazione, procre- 
azione e, infine, morte. Lo svelamento del nudo, dell'atto sessuale e dei suoi organi e, contemporaneamente, le forme per favorire l'approssimazione fra i due sessi (il Decameron è, in fondo, un manuale dell'arte della seduzione) rispondono a un principio di assoluta visibilità, un movimento analogo a quello della pittura dell'epoca. Si può dire che il Decameron sia un testo osceno, non tanto perché l'atto sessuale è il vero fine e protagonista di molte avventure, quanto perché allude a una possibile etimologia della parola: a ciò che è $o b$-scena, cioè che è possibile far discendere dal latino $o b$-scaena, "fuori dalla scena". Non certamente al di fuori della vita, ma trasbordando largamente i limiti della letteratura erudita del tardo Medioevo, dei suoi modelli e riferimenti espliciti. La vera e propria pornografia viene evitata, perché il vorticoso e iperbolico intreccio di corpi, classi e funzioni sociali raramente avviene all'insegna del compenso in denaro, che caratterizzerebbe un "un parlar di puttane”. Anticipando le critiche, la novella di Filippa (VI, 7) descrive la burla del seduttore ai danni di una donna troppo facile. In questa novella il meretricio, il compenso per l'atto sessuale è trasformato furbescamente in restituzione di un prestito. L'erotismo del mondo viene così immesso con forza nel mondo nuovo della letteratura. Boccaccio fonda una nuova idea della letteratura (il racconto, la novella) che si basa sull'erotismo differito o sblimato: esteso al rapporto fra lettore e testo, qualcosa che anticipa in senso vagamente borgiano sia Roland Barhes (Framenti di un discorso amoroso) che Sigmund Freud (Al di là del principio del piacere), nel senso di una sublimazione dell'erotismo, con l'effetto di una seduzione del lettore da parte del testo. Ogni novella possiede un dentro e un fuori, una visione che viene da prospettive incrociate, che testimonia una ricerca análoga a quella delle tendenze contemporanee della pittura: abbandono dei modelli simbolici medievali, per inserire il contesto storico dentro all'immagine descritta, mettendo a fuoco la prospettiva dei protagonisti e il loro sfondo sociale e culturale. L'amore, il desiderio, la seduzione sono affrontati con estrema delicatezza, con una punta sempre maliziosa, ma mai apertamente volgare, con una sottile vena d'ironia ed un allegro distacco. Il testo è ironico perché afferma e nega, allo stesso tempo, fa balenare la seduzione, l'erotismo, la pornografia per poi negarli e usarli come esempi del contrario: metafore del testo, vaghe allusioni. Non si tratta solo di ambiguità, ma di vera e propria tendenza elusiva, come è già stato affermato da Mazzotta nel eccellente libro The world at play, ${ }^{*}$ forse

* (MAZZOTTA, Giuseppe. The world at play in Boccaccio's Decameron. Princeton: Princeton Univ. Press, 1986.) 
* (BOCCACCIO, Giovanni, op. cit., Vol II , "Intr. IV Giornata": 459-60.) la migliore visione contemporánea del Decameron. Il testo, quindi, afferma e nega, allo stesso tempo. É realisma e il suo contyrario, in maniera più che paradossale.

Ciononostante il Decameron è stato sottoposto a una sequenza di critiche, fin da quando l'autore era ancora in vita, arrivando a essere messo all'indice, fra i libri proibiti, al tempo del tribunale dell'Inquisizione. Ancora oggi il suo erotismo può sorprendere per la spregiudicatezza. Espressioni crude e frasi eleganti, un pastiche, indicano un gusto per la mescolanza di stili: umile e sublime, giustificata dalla caratterizzazione di personaggi e situazioni socialmente differenziati. È un dipinto con una scena totale: il tardo Medioevo che si affaccia sulla modernità, ma già la prefigura, ne abbozza lo stampo. Ciononostante l'understatement è esplicito. Le novellette (non le novelle) sono scritte in stile umilissimo e rimesso:

le presenti...novellette..., le quali non solamente in fiorentin volgare e in prosa scritte per me sono e senza titolo, ma ancora in istilo umilissimo e rimesso quanto il più si possono. ${ }^{* 9}$

La rappresentazione è scanzonata e trasparente. Il rapporto sessuale viene descritto come espressione di una legge della natura, che Filippo Balducci, protagonista dell'introduzione della IV giornata, svelerà come regolatrice dei rapporti: "sentì incontanente più aver di forza la natura che il suo ingegno"; qualcosa di molto forte che scardina le convenzioni sociali, religiose e culturali dell'epoca. In effetti, Filippo Balducci rompe lo schema delle 100 novelle (secondo il criterio dell' elusività affermato da Giuseppe Mazzotta) e in pratica presenta una novella il cui numero è 101; Giovanni Boccaccio, pertanto, sarà l' $11^{\circ}$ narratore, una forma di esplodere la macchina narrativa appena enunciata. In fondo il Decameron, come più tardi L'amante di Lady Chatterley (il cui autore D. H. Lawrence era un grande lettore di Boccaccio), sono testi che lodano liberamente l'apertura verso il sesso., e in questoil Decameron è ecrtamente un testo precursore.

Nella prima sezione il sesso è sempre accomunato al clero: dal fraticello, all'abate, dalla monaca alla badessa; la topografia delle avventure illumina la vita di conventi, monasteri, eremitaggi,

\footnotetext{
9 "Terei disto a prova cabal nas presentes novelas, redigidas por mim em florentim vulgar e em prosa, sem título algum e ainda no estilo mais humilde e sóbrio de que posso dispor." (BOCCACCIO, Giovanni. Decameron. U. Tavares Rodrigues (trad.). Belo Horizonte: Crisalida, 2005: 225.)
} 
focalizzando situazioni più che scabrose. L'ipocrisia della retorica religiosa viene svelata, denudata letteralmente. Esiste, però, un elemento più profondo, che provoca un intenzionale sovrapposizione fra rituale religioso e rituale sessuale. Un ritorno e una rivalutazione del corpo, con un'intenzione, nel suo effetto finale, per niente oscena, anzi molto concreta e produttiva. L'affermazione di un nuovo culto, retto da regole precise, base di una nuova etica che, in una certa misura, coincide con quella del testo: l'etica della seduzione, dell'approssimazione, del congiugimento, della còpula. Quest' ultima, anche per Freud, è espressione dello spirito della vita, dell'eros, che si contrappone all'appiattimento, al livellamento, alla pesantezza, alla pedanteria, allo spirito della morte, o tanatos. ${ }^{10}$

\section{La parodia, la beffa, I'equivoco, lo scambio}

L'architettura stessa, che regge un maestoso edificio geometrico (dieci giornate, cento novelle, con simmetrie e giochi di specchi), si svela facilmente come parodia tramite un'allusione manifesta alla Divina Commedia, ma, evidentemente, con tutt’altro propósito rispetto al capolavoro del illustre e incombente predecessore:

Questo orrido cominciamento vi fia non altramenti che a' camminanti una montagna aspra ed erta, appresso alla quale un bellissimo piano e dilettevole sia riposto. ${ }^{* 11}$

Il titolo originale del testo suona: Decameron, cognominato prencipe Galeotto e contiene due rimandi espliciti: l'Hexameron di Sant'Ambrogio (339/40- 397), una visione dei sei giorni della creazione (che Boccaccio trasforma in dieci). Il sottotitolo richiama un episodio (recente all'epoca di Boccaccio, nato quando Dante era ancora in vita), ma già famoso: la storia di Paolo e Francesca, descritta nel V canto dell'Inferno, storia tragica e sublime di due giovani letteralmente traditi da un libro e indotti dall'esempio di Lancelot e Ginevra, il cui incontro venne facilitato da Gallehaut, l'intermediario. "Galeotto fu il libro e chi lo scrisse", dirà France-

\footnotetext{
10 "La cópula, l'unione di due organismi unicellulari, ha l'effetto di tenere in vita e ringiovanire entrambi." (FREUD, Sigmund. Al di là del principio del piacere. Int. Psychoan. Verlag. Leipzig/ Wien/ Zürich, 1921: 49.)

11 "O pesadelo do princípio? Imaginai uma montanha cujas escarpas tenham surgidodiante dos viajantes, junto dela, porém, há uma planície tanto mais bela e sedutora quanto maior for o cansaço." (BOCCACCIO, Giovanni. Decameron. U. Tavares Rodrigues (trad.). Belo Horizonte: Crisalida, 2005: 15.)
} 
* (Anônimo. Il Parnasso italiano continuato: Boiardo, Buonarroti, Tassoni, Boccaccio. Lipsia: Ernesto Fleischer, 1833: 1815: XIV.) sca al pellegrino Dante. In un'antica edizione del Decameron l'anonimo commentatore sapientemente scrive:

Galeotto. I Deputati credono questa voce presa da Dante nel V dell'Inferno. ... Leggi la nota sopra questa voce nel suddetto libro, e troverai che il Glosatore lo interpreta Seduttore, o sia Mezzano d'amore.*

Il Decameron si presenta così come un libro che richiama la Divina Commedia , ma ne capovolge lo spirito: è un libro seduttore, in cui obiettivo non è più l'elevazione dell'interlocutore (o ascoltatore). "Ogni storia è l'occasione per una nuova storia”, perché le novelle sono intrecciate l'una all'altra e, allo stesso tempo un secondo movimento si proietta al di là del testo, verso l'attività della lettura e dell'interpretazione. Nella sua operazione di smontaggio delle credenze e illusioni (la religione, le professioni considerate di rilievo, i modelli dell'amore distaccato e platonico, l'ideale della bellezza e della morale assolute) il testo funziona come una macchina, il cui fine è la letteralizzazione delle metafore. ${ }^{12}$

La mescolanza degli stili è destinata anche mimeticamente a mostrare i rovesciamenti costanti delle situazioni. In questo mondo concreto, palpabile, la lotta per la sopravvivenza è descritta nella sua opposizione all'idea tradizionale del destino, del fato, della fortuna classica e medievale, cioè un tracciato sostanzialmente scritto in precedenza (dagli dèi pagani o dalla religione cristiana); l'iniziativa dei personaggi delle novelle si basa su una nuova arma: l'ingegno, la parola, strumento privilegiato per mettere in scacco la provvidenza cieca o ingiusta. Una sottile filigrana di parole o motti, provoca nel testo continui capovolgimenti che, nella loro ingegnosa costruzione, mettono a nudo una rete di equivoci, di scambi, di beffe: un flusso continuo di smontaggio e rimontaggio, che rappresenta mimeticamente un mondo in movimento, descritto in maniera realistica. L'arte della narrazione del Decameron sta in questo apparente fluire che si organizza in una precisa geometria, con rimandi e giochi di specchi che interagiscono e che ne rendono la lettura infinita.

\footnotetext{
${ }^{12}$ A este breve anojamento (chamo-lhe breve porque ocupa poucas linhas) sucedem-se logo a doçura e o prazer (BOCCACCIO, Giovanni. Decameron. U. Tavares Rodrigues (trad.). Belo Horizonte: Crisalida, 2005: 15)

"The gesture is understandable, but it would be justified only if it where accompanied by the critical awareness of the elusiveness and permanent displacement that the metapher of play governing the Decameron engenders". (MAZZOTA, G. The world of play. Princeton. P.Univ. Press., 1986: 12.)
} 
A metà esatta dell'originale (la cinquantunesima novella VI, 1) si trova una metanovella che si potrebbe intutolare "l'arte della parola". Lì si narra l'incapacità di un cavaliere nel raccontare una novella. Si tratta, in sostanza, di una falsa novella, totalmente inesistente, oppure - meglio - una metanovella. É la IV giornata dell'originale ad apririsi con un racconto del narratore (il cui nome è Giovanni Boccaccio, casualmente uguale a quello dell'autore e quello del protagonista) che narra la novella di Filippo Balducci, decisiva per la sua "morale" e che scardina completamente il conteggio, mettendo in discussione la geometría del numero 100 (geometría che rimetteva a Dante e allusione ai cento canti della Divina Commedia. Il narratore denuncia - insolitamente - che questa sua novella sarebbe sorprendentemente incompleta, difettosa rispetto alle altre novelle alle quali non si vuole mescolare:

acciò che non paia che io voglia le mie novelle con quelle di così laudevole compagnia...mescolare. ${ }^{* 13}$

* (Ibidem: 462.)

A seguito della morte dell'amata moglie e, volendo sottrarre il figlio dall'influenza morale nefasta della città, Filippo Balducci (protagonista di questa novella anomala) si ritira a vivere con il figlio in eremitaggio. Alla prima visita alla città di Firenze, quando il figlio diviene diciottenne, cerac invano di distoglierlo dalla vista delle "belle giovani donne e ornate". Al contrario, il figlio ne viene fatalmente attratto e afferma rapito: "Elle sono più belle che gli agnoli dipinti". Filippo tenta di lottare contro questa impressione: "elle son mala cosa", o almeno impedirgli una corretta identificazione delle donne: "Elle si chiamano papere", mente. Ma questi tentativi maldestri non diminuiscono il desiderio del figlio, che chiede di portarne una con loro, per darle da beccare. La metafora sessuale e ironica è chiarissima:

Deh! se vi cal di me, fate che noi ce ne meniamo una colà su di queste papere, e io le darò beccare. ${ }^{* 14}$

Il narratore mette in bocca al figlio di Balducci tutta la sua ironia, dove il beccare ha un esplicito significato allusivo. La novella svela, dunque, la legge della natura, cioè l'istinto sessuale come vero

\footnotetext{
${ }^{13}$ Não quero misturar as minhas próprias histórias às da nobre companhia que vos apresentei, mas um simples fragmento de historieta, cujo caráter inacabado vos mostrará por si próprio que não tem a mesma origen. Boccaccio:2005: 225,6

${ }^{14}$ Se vos preocupais comigo, fazei com que possamos levar lá para cima uma dessas passarinhas. Eu lhe darei de comer . Boccaccio: 2005:227
} 
* (BOCCACCIO, Giovanni. Elegia di Madonna Fiammetta. Milano: Mursia, 1978: 14.) e proprio motore della vita: "[Filippo] sentì incontanente più aver di forza la natura che il suo ingegno". Con questa frase il Decameron anticipa secoli. Trova la sua legge della natura, diviene il portavoce del progresso della libertà. La novella, come affermato, viene dichiarata inconclusa dal narratore:

mi piace in favor di me raccontare, non una novella intera, acciò che non paia che io voglia le mie novelle con quelle di così laudevole compagnia... mescolare, ma parte d'una, acciò che il suo difetto stesso sé mostri non esser di quelle. ${ }^{* 15}$

Excusatio non petita? No: si tratta di un vera e propria frode del narratore! Questa novella dislocata, fuori posto, una novella eccessiva, crea una contrapposizione fra l'ipernarratore (Giovanni Boccaccio) e i dieci novellatori 'regolamentari' delle dieci giornate (sette donne e tre ragazzi). Ed è tramite questa novella più che da qualsiasi altra che emerge una dichiarazione ideologica del testo. Possiamo concludere con le parole de L'Elegia di Madonna Fiammetta, in cui la finzione viene esplicitata:

Io, semplicissima giovane e appena potente a disciogliere la lingua nelle materiali e semplici cose tra le mie compagne, con tanta afflizione li modi del parlare di costui raccolsi, che in brieve spazio io avrei di fingere e di parlare passato ogni poeta; e poche cose furono alle quali, udita la sua posizione, io con una finta novella non dessi risposta dicevole*16

La novella dà un senso compiuto del mondo del narratore e mostra la "legge della natura": l'eros, che il libro nel suo insieme rivela. Di conseguenza, come si vedrà, senza il narratore $i$ conti non tornano. Avremo una vera e propria sovversione del principio architettonico enunciato: sottraendo la novella che abbiamo chiamato "l'arte della parola" (la novella di Madadma Oretta, VI, 1) già menzionata, si avranno solo 99 novelle. Aggiungendo la novella che abbiamo definito "dell'ipernarratore \Giovanni Boccaccio" (Intro-

${ }^{15}$ Quero defender-ma a mim próprio contando-vos não uma novela completa - não quero sequer parecer misturar as minhas próprioas histórias às da nobre comppanhia que vos apresentei - mas um simples fragmento da historieta, cujo caráter inacabado vos mostrará por si próprio que não têm a mesma origen (Boccaccio: 2005. 225-6)

${ }^{16}$ Eu jovem simplerrima, com apenas o poder de apresentar uma língua das coisas materiais e simples entre minhas amigas, com tanto cuidado prestei atenção à maneira de falar dele, que em pouco tempo eu deveria fingir e falar como os poetas; e para poucas, que ouviram minha posição, com uma novela falsa não conseguisse dar uma resposta adequada. Boccaccio: 1978:14, trad minha. 
duzione alla IV giornata), una novella più che completa e contiene l'ideologia fondamentale del Decameron, cioè la sua "scoperta" che existe una "legge della natura più forte della volontà umana, si avranno, a seconda dei casi: 101 o 100 novelle. Ma i narratori (con l'aggiunta del meta o ipernarratore) saranno divenuti undici e non più dieci. Affermazione ed elusione del principio della costruzione, quasi che il testo volesse mettere a fuoco una crepa, svelandoci un edificio imperfetto, incompiuto, parziale, ma, allo stesso tempo: stimolante, aperto, proiettato.

\section{La nascita del narratore}

Il narratore Boccaccio nega a se stesso il ruolo di autore, creatore e demiurgo del genere della novella, e afferma aver ripreso dalla tradizione i suoi racconti: "io non poteva né doveva scrivere se non le [novelle già] raccontate".* Il Decameron si presenta, pertanto, como un'operazione di rilettura e riscrittura di materiale già noto. In effetti, la maggioranza dei temi svela modelli e situazioni affermate (da L'asino d'oro di Apuleio alle Metamorfosi di Ovidio, al Satyricon di Petronio), con l'inserimento di un vivace contesto degli episodi di cronaca del tempo di Boccaccio, alcuni racconti orientali che verranno a aessere assemblati molto più tardi con il titolo le Mille e una notte, ma all'epoca erano conosciuti col nome il Libro dei Sette Savi; ci sono anche spunti da Il Novellino, una raccolta anonima di brevi storie e aneddoti, di alcuni decenni precedebnte. L'illusione realistica costantemente affermata viene così sostanzialmente negata: i riferimenti sono prevalentemente letterari e il narratore Boccaccio si presenta, all'inizio della nostra tradizione letteraria moderna (o neolatina), come semplice intermediario, non più un classico della letteratura, ma un artigiano della parola, espressione del nuovo mondo che descrive. La profonda ironia del testo sta nella polarità fra affermazione e negazione: un mondo descritto in maniera realistica e palpabile che scopre l'artificio della parola e anticipa la letteratura come menzogna.

Architettura e realismo sono il prodotto di un nuovo genere, il cui ritrovato fondamentale non è tanto o solo quella della cornice (elemento giustamente valorizzato dalla storia della letteratura): legame fra vari racconti, strategia generale. Ma è la presenza nuova, esplicita e marcante, della nuova funzione del narratore l'elemento più moderno. Boccaccio, al contrario di Dante e di autori a lui 
precedenti, entra in scena esclusivamente come narratore, con un understatement della propria funzione: non più creatore e demiurgo, ma interprete, rilettore e redattore di un testo. Questa posizione è attestata in tre momenti strategici, nella struttura del Decameron: nel Proemio, nell' Introduzione alla I giornata, dove si racconta della finalità del libro e della peste a Firenze, e nella Conclusione "dell'autore". Il narratore Boccaccio stabilisce il parallelo già citato fra letteratura e pittura, rivendicando alla prima la libertà di descrivere il nudo, cosa che la seconda già faceva da tempo. Appare qui una vera e propria finalità del testo: mostrare non solo la giustapposizione delle due arti, quanto la necessità che la pittura ha di appoggiarsi sulla letteratura: necessità di un artista poliedrico e colto, che si rivolge a un pubblico di lettori, sia esso di pittori o di letterati, capovolgendo - in anticipo - la nota posizione di dipendenza della letteratura dalla pittura, che verrá affermata nel Rinascimento. Il narratore Boccaccio crea dieci narratori che, raccontandosi delle storie, formano un pubblico che commenta le novelle, istituendo vari piani del racconto: il piano del narratore, il piano del pubblico dei narratori e, infine, il piano dei personaggi secondari di ogni storia e così via, in una progressione vorticosa che ricorda quello delle delle scatole cinesi. Le cornici concentriche, che rispondono al criterio architettonico, istituiscono una tensione decisiva fra il narratore (o i narratori) e il loro pubblico, in una dialettica crescente fra interno e esterno, fino a includere noi lettori contemporanei. Se l'erotismo è uno strumento di avvicinamento decisivo alla letteratura, innalzando la commedia umana a tema di interesse universale, l'inclusione del lettore e del pubblico (tramite la proliferazione del commento vivo ai racconti), crea un tipico gioco di specchi in cui, a partire da Boccaccio, nel testo vedremo principalmente la nostra immagine, la nostra genealogia. Letto in questa prospettiva il Decameron sfata due paradigmi considerati ancora recentemente validi in letteratura: l'illusione realista ${ }^{17}$ e il mito dell'autore. Nel primo caso il testo si mostra come prodotto di una strategia per catturare il lettore ("le già dette donne, che quelle leggeranno"); nel secondo caso, il ribassamento dell'autore a mero riproduttore, intermediario e traduttore di testi ripresi dalla tradizione, rende inutile una

${ }^{17}$ Più che al testo di Michel Rifaterre ("A ilusão referencial”. In: Literatura e realidade. O que é o realismo. Lisboa: Dom Quixote, 1984: 102) qui si immagina un'illusione realista o naturalista che confonde il testo letterario con un testo comunicativo o storico, inteso in senso letterale. 
ricerca della personalità dell'autore, all'interno del testo (lo scrittore, che non fui):

se pur presupporre si volesse che io fossi stato di quelle e lo 'nventore e lo scrittore, che non fui, dico che non mi vergognerei che tutte belle non fossero, per cio' che maestro alcun non si truova, da Dio in fuori, che ogni cosa faccia bene e compiutamente... ${ }^{* 18}$

Alla virtù maieutica della poesia e del teatro corrisponde in Boccaccio un'arte profondamente laica, cioè non legata all'etica attraverso il filtro della filosofia o della religione, bensì un'etica della scrittura o, meglio, della lettura derivante dalla funzione performativa del discorso, dalla pragmatica del quotidiano. Lo sfondo realistico è sempre giustapposto a una trama deformata, sostanzialmente inverosimile: estrema, comica o grottesca. Il suo realismo sta nel riconoscimento di uno statuto nuovo del discorso, dell'arte della parola, dell'erudizione: non più alla ricerca del sublime o dell'intrattenimento, ma in bilico fra i due campi. Strumento di uso quotidiano dei mercanti, degli avventurieri, del loro milieu culturale, cioè di una nuova classe. Si potrebbe concludere con un paradosso: la nostalgia del narratore, di cui parla Benjamin nel suo famoso saggio su Nicola Leskow, ${ }^{*}$ di fatto potrebbe alludere a Boccaccio che, contrariamente a quanto si poteva inizialmente supporre, mostrava in germe tutta la potenzialità della scrittura. Il narratore è etimologiamente "colui che è informato" e (rac)conta le sue novelle. La strategia del narratore nel Decameron indica un malizioso gioco di rimandi fra il narratore Boccaccio, i dieci narratori e il loro pubblico (come già mostrato). Forse l'erotismo del testo, il realismo e l'architettura del Decameron centrano esclusivamente una vera grande beffa: quella ai danni del lettore, maliziosa trovata che rende il Decameron ancora un libro a noi contemporaneo.

La morte, la culinaria

La cornice esterna ai racconti è rappresentata da una descrizione crudele e meticolosa degli effetti fisici, umani e morali della peste. Più d'una allusione fa capire che il narratore è abbastanza

\footnotetext{
${ }^{18}$ Mesmo admitindo - o que não corresponde à verdade - que eu seja ao mesmo tempo o autor e o escritor, claro que não iria corar pelo fato de nem todas as histórias serem belas. Que criador - incluindo Deus - realiza uma obra prima absoluta? (Boccaccio: II: , tradução minha)
}

* (BOCCACCIO, Giovanni, op. cit., II: 1258.)

* (BENJAMIN, Walter. "II narratore". In: Angelus Novus. Saggi e frammenti. Torino: Einaudi, 1962: 247.) 
* (BOCCACClO, Giovanni. Decameron. V. Branca (org.). Torino: Einaudi, 1992: 18.)

* (BOCCACCIO, Giovanni, op. cit., Vol I, "Introduzione alla I giornata": 27.)

* (Cf. MAZZOTA, op. cit.; BATTAGLIA RICCI, Lucia, op. cit.)

* (SONTAG, Susan. A doença como metáfora. São Paulo: Companhia das Letras, 2007.)

* (PAGLIA, Camille. Personas Sexuais. São Paulo: Companhia das Letras, 1990.)

* (CALVINO, Italo. II visconte dimezzato. Torino: Einaudi, 1984.) scettico sull'origine divina della epidemia, poiché questa origina dlle paure e immaginazioni:

Dalle quali cose e da assai altre a queste simiglianti o maggiori nacquero diverse paure o immaginazioni. ${ }^{* 19}$

La peste, insomma, è un evento reale catastrofico, poiché decima la città di Firenze e buona parte d'Europa ("oltre a cento milia creature umane si crede per certo dentro alle mura della città di Firenze essere stati di vita tolti",* ossia i 9/10 dei suoi abitanti originari. Allo stesso tempo, la sua descrizione, con toni iperrealistici, segue schemi letterari già noti, anche se in altri contesti: la descrizione della peste in Atene di Tucidide, ma anche le analoghe (e differenti) descrizioni di Lucrezio, Galeno, Ippocrate, Gregorio di Tour, Paolo Diacono o di Sant'Agostino.* Se la peste è stata vista sempre come una malattia demoniaca e fatale, legata all'ira divina, come nel caso di Edipo Re, perché un'inizio tanto tragico, per un libro, la cui principale funzione esplicita era il mero intrattenimento?

Difatto la peste, di tutte le malattie, è la più radicale, assoluta, quella che meglio simbolizza un mistero osceno, cioè quello della norte, ${ }^{*}$ che mette a nudo il corpo nella sua oscenità* che favorisce la trasgressione. ${ }^{*}$ In ogni caso mostra l'impossibilità di un contenimento etico del mondo reale. Ma la morte non è solamente l'estremo confine di questo mondo, di cui scandisce il tempo che scorre, rappresentandone il limite estremo, è anche qualcosa che può essere descritto e superato solo metaforicamente, tramite la letteratura.

L'analogia fra superamento della morte tramite la letteratura - un aspeto simbolico - e il superamento delle colonne d'Ercole da parte dei navigatori tardo medievali - un passo concreto - trova nel Decameron una sua compiuta e chiara affermazione. Il Decameron appare come una legittima riscrittura dell' Hexameron di Sant'Ambrogio, non nel senso edificante degli exempla medievali, ma nel suo contrario: l'affermazione di un mondo del possibile, del narrabile, un mondo che, come quello biblico, si aprirà tramite il verbo. La descrizione realistica della peste tende a sfatare una sua visione allegorica (come punizione) letteralizzandola nella sua descrizione

\footnotetext{
${ }^{19}$ Esses accidentes e muitos outros do mesmo gênero, senão piores, fizeram nascer, naqueles que continuavam vivos, pânicos e obcessōes de diferentes espécies... Boccaccio: 2005: 17
} 
crudamente realistica. L'ironia del testo sta nel mostrare come il diabolico non deve essere visto dal punto di vista dell'interpretazione allegorica (e canonica), ma molto più vicino ai nostri occhi: debe essere indagato nel suo effetto sul corpo. In fondo un'amore per il corpo (che la contemplazione della norte mette in moto) che suggerisce uno scambio fra morte e amore, come paronomasia.

C’è da chiedersi, forse, perché a partire da queste considerazioni così tragiche sia nato un libro pieno di vigore e ottimismo come il Decameron, all'inizio di un millennio che si è chiuso con la tragedia dell'Aids, flagello simbolicamente altrettanto terribile quanto la peste e che ha favorito una chiusura conservatrice e moralista, all'insegna della "punizione per aver vissuto una vita insana" (S. Sontag). La risposta sta, forse, in quello che è stato affermato a proposito dell'Aids: "la malattia ha creato un altro individuo", arrivando al paradosso che è opportuno considerare l'ironia come un valore al di sopra di qualsiasi altro. Ma prendere quest'affermazione ironicamente.

\section{La cura della parola}

Analizzando il Decameron, una sezione a parte dovrebbe essere dedicata all'arte della parola. La novella più significativa, dopo quella di Madama Oretta (VI, 1), è quella dedicata a Giotto (VI, 5), "il primo maestro del naturalismo in Italia",* il grande pittore, contemporaneo di Boccaccio, la cui produzione impone una visione razionale e semplificatrice della realtà. Difatti, il nucleo della novella è centrato sul rapporto fra apparenza e sostanza, fra bruttezza fisica e sublimità artistica e mostra la necessità di un artista pieno, in grado di dominare la retorica e arte della pittura, o meglio: di poter dominar quest'ultima solo grazie alla prima. Il duello verbale (VI, 5) con Messer Forese da Rabatta, anche lui descritto come la quintessenza della bruttezza ("essendo di persona piccolo e sformato, con viso piatto e ricagnato..."), mostra un Giotto edotto nell'arte della parola, "bellissimo favellatore". Sembra come se il narratore volesse capovolgere una remoto senso del vedere, qualcosa che la sostanza etimologica della visione trasmette: se infatti vedere viene dalla radice indeuropea WEID, ${ }^{*}$ che significa "ho visto e quindi so", il testo ci mostra che per vedere realmente occorre sapre, dominare l'arte della nuova retorica, quella della battuta, del Witz, una parola tedesca che deriva da Wheid e che, allo stesso tempo, include ironia
* (HAUSER, Arnold. Storia sociale dell'arte. Torino. Einaudi: 2001.)

*(DEVOTO, Giacomo. Avviamento all'etimologia italiana. Milano: Mondadori, 1979.) 
* (RIPA, Cesare. Iconologia. Milano: Tea, 1992.)

* (BOCCACCIO, Giovanni, op. cit., Vol I, "Proemio": 9)

* (BOCCACCIO, Giovanni, op. cit., Vol II: 719.) e sapere. In questo Boccaccio cerca di enfatizzare nella sua lettura dell' ut pictura poesis, una certa prevalenza della letteratura rispetto al precetto classico, derivato da Orazio, secondo il quale pittura e letteratura dovevano essere considerate allo stesso livello, tema che Cesare Ripa nel Seicento aveva riptreso, capovolgendolo: "la poesia tace nella Pittura \& la Pittura nella poesia ragiona".* Boccaccio anticipa e contribuisce a creare le premesse del Rinascimento, ergendosi contro la tradizione delle arti classiche (tra trivium e quadrivium), e afferma, implicitamente, una nuova triade di arti: le arti figurative (pittura e scultura), architettura e letteratura. Le vecchie professioni (medicina, giurisprudenza, clero) vengono ribassate, o meglio, private dell'aura che le contraddistingueva. Si afferma, al contrario, il principio di una nuova grammatica, quella della novella, basata sulla costruzione e sulla visibilità. Il flusso delle avventure scorre libero, fluente, vivace e ricco, senza soste: un frenetico rincorrersi dei protagonisti nella loro vita quotidiana fatta di "udire e veder molte cose, uccellare, cacciare, pescare, cavalcare, giucare o mercatare":* non più cavalieri e dame medievali, ma protagonisti a cavallo del testo, una metafora che si svelerà nella sua ambiguità, come ambigua può essere la lettura della stessa Madama Oretta (VI, 1), portata a cavallo dal balbettante novellatore impotente.

In questa telegrafica novella tutto è centrato sull'arte del racconto, una storia sulla storia: più che raccontare una storia, racconta di come la storia, mal raccontata, cessa il suo effetto e provoca nella protagonista una crisi drammatica: "udendolo, spesse volte veniva un sudore e uno sfinimento di cuore, come se inferma fosse stata per terminare"* La velocità delle scene è cinematográfica e ricorda uno dei primi film del XX secolo, dove le azioni dei personaggi si svolgono rapide e ci danno il sapore della freschezza dell'origine ed il Decameron come un tutto appare come un libro effettivamente fatto di movimento, laddove la Divina Commedia tendeva a cogliere un'istantanea essenziale, una figura, come una fotografia. La lunga novella di frate Cipolla (VI, 10) è nuovamente destinata a mostrare gli effetti del discorso: una folla credulona è raggirata in mille modi tramite il discorso menzognero del frate. Possiamo però considerare la prima novella, la novella di apertura, una chiusura in questo piccolo panorama. Si trata di un capolavoro di ambiguità, introduzione alla macchina narrativa. Ser Ciappelletto $(\mathrm{I}, 1)$ realizza in punto di morte, davanti agli occhi stupiti dei suoi ospiti, una metamorfosi radicale della propria figura: $\mathrm{da}$ 
mentitore, truffatore e ladro a figura esemplare di santo in grado di provocare, dopo morto, addirittura miracoli. Il meccanismo della trasformazione avviene tramite una confessione, che solo i suoi ospiti e il narratore sanno essere falsa. L'accento principale del testo è sul verbo credere:

credeva esser vero ciò che ser Ciappelletto avea detto: e chi sarebbe colui che nol credesse, veggendo uno uomo in caso di morte confessandosi dir cosi?*20

La metamorfosi sarà completa, grazie a una distorsione in francese del cognome originale del personaggio (ser Cepparello), in Ciappelletto, la cui origine etimologica va ricercata in cappello, oppure ghirlanda: giustificazione, a posteriori, della sua aura, adeguata alla sua fama. In questo il Decameron mostra la sua natura classica: un libro che a una prima lettura appare singolarmente già conosciuto (tramite le infinite nuove versioni e imitazioni, le parodie, le commedie, e libri, film) e che una nuova lettura mostra in luce sorprendentemente nuova.

Se il diavolo in corpo svela un diavolo del testo e nel corpo, il testo sconvolge anche l'illusione di una morale canonica, ufficiale. A prima vista è la legge della natura, l'erotismo, che prevale. Il cappello o l'aura legano idelamente, in questa selezione, la prima e l'ultima novella: in quella Masetto, finto sordomuto, alla fine della sua carriera torna al suo paese contento di aver "posto a Cristo le corna sopra 'l cappello".* Forse una sottile allusione all'aura di Ciappelletto e che fa della morale una questione di corna. La lotta fra bene e male è qui ridotta a parodia. Griselda, protagonista dell'ultima delle cento novelle, trasforma la sua iperbolica virtù in cinismo crudele e mostra che non c'è salvazione, specialmente non nella moralità bigotta e radicale. Forse un'indicazione che l'etica del mondo è troppo viziata dalla retorica. Forse un preannucio che l'etica, quella nuova, va cercata nel testo.

Abbiamo cominciato con un rovesciamento (il diavolo) e terminiamo con un'autoironia (Ciappelletto). L'ordine è stato arbitrariamente sconvolto. Emerge da un lato, il piacere del testo e, dall'altro, la sua elusività, una somma di incertezze propositali. Ciò che è (il realismo) viene svelato come non del tutto pertinente.

\footnotetext{
${ }^{20}$ Olhava-o como ao mais santos dos homens, visto acreditar completamente no que dissera. E quem não acreditaria, ouvindo tais palavras na boca de um moribundo ? Boccaccio: I: 36
}

* (BOCCACCIO, Giovanni. Decameron. V. Branca (org.). Torino: Einaudi, 1992: 66.)

* (BOCCACCIO, G., op. cit. Vol II , III, 1, p. 337.) 
Ma la messa in scena dello svelamento, il testo lo mostra, provoca una modifica di ciò che è. Il risultato è una trasformazione, uno scambio, una beffa. Una modifica della realtà, mentre si afferma l'incapacità di rappresentarla, il vizio della visione.

$\mathrm{Ma}$ è solo la letteratura che trascende il puro contesto, il destino, il caso, la fortuna. La morale (quella tradizionale) di fatto non esiste. L'etica in letteratura non è che la lettura della letteratura. Un'altra etica sorge solo quando si rimira la morte: qualcosa che è totalmente fuori dal contesto, fuori dal mondo (da questo mondo). La nuova etica del testo è la ricerca dell'effetto ingegnoso, arguto, vivace, intelligente, spiritoso. L'affermazione, e il suo contrario. Appunto. La letteratura come arte e finzione.

Andrea Lombardi é professor de literatura italiana na UFRJ. Após um diploma prematuro em Música (conservatório de Nápoles), formou-se em Letras na Universidade de Roma. Tomou a decisão radical de emigrar para o Brasil, por achar que o velho mundo carecia de energias para resolver seus problemas, sobretudo éticos. Foi leitor na UFRGS e depois na USP, onde começou um doutorado «bloomiano» pelo título: «Calvino, Ariosto e a influência poética». Lecionou literatura italiana na USP e em 2005 mudou-se com sua família para Niterói, achando a proximidade com o mar mais saudável. Email: <lombardi.andrea@gmail.com> 